\title{
Maternal factors, ultrasound and placental function parameters in early pregnancy as predictors of birth weight in low-risk populations and among patients with pre-gestational diabetes
}

\author{
Anna Gasiorowska ${ }^{1}$, Agnieszka Zawiejska², Piotr Dydowicz ${ }^{3}$, Ewa Wender-Ozegowska², \\ Grzegorz Poprawski ${ }^{4}$, Kinga Tobola-Wrobel ${ }^{5}$, Katarzyna Ziolkowska ${ }^{6}$, Marek Pietryga ${ }^{3,5}$ \\ ${ }^{1}$ Gynecology and Obstetrics Ward, Podhalanski Specialist Hospital, Nowy Targ, Poland \\ ${ }^{2}$ Department of Reproduction, Poznan University of Medical Sciences, Poland \\ ${ }_{3}^{3}$ Prenatal Diagnostic Center, Gynecology and Obstetrics Hospital, Poznan University of Medical Sciences, Poznan, Poland \\ ${ }^{4}$ Chair and Clinic of Oncology, Poznan University of Medical Sciences, Poznan \\ ${ }^{5}$ Department of Obstetrics and Women's Disorders, Chair of Gynecology, Obstetrics and Gynecological Oncology, \\ Poznan University of Medical Sciences, Poznan, Poland \\ ${ }^{6}$ Chair and Department of Laboratory Diagnostics, Poznan University of Medical Sciences, Poznan, Poland
}

\begin{abstract}
Objectives: The aim of our work was to assess the usefulness of maternal factors, ultrasound and placental function parameters during early pregnancy as predictors of birth weight in populations of healthy pregnant women and women suffering from pregestational diabetes.

Material and methods: A study group comprised 97 healthy women and 160 women with pregestational diabetes (PGDM, type 1), all in singleton pregnancy. Ultrasound examination was performed between weeks 11 and 14 , and in weeks 20 and 30 of gestation, based on recommendations of the Polish Society of Gynecologists and Obstetricians, Ultrasonography Division. We also checked uterine artery blood flow parameters. During the first trimester consultation, all patients were surveyed and the following data were collected: age, BMI, reproductive history, comorbidities and smoking. We also collected blood samples and assessed PIGF, PAPP-A, and BhCG levels.

Results: Our study showed that newborn birth weight negatively correlated with mother's age, her diastolic blood pressure, PI of her uterine arteries and BhCG protein levels. Moreover, birth weight directly correlated with PIGF and PAPPA-A protein levels, and maternal early-pregnancy BMI.

Conclusions: LGA diagnosis in the first trimester of pregnancy allows for selection and modification of some risk factors and closer monitoring of endangered fetuses throughout the pregnancy, with emphasis on the perinatal period.

Parameters with confirmed usefulness in the prediction of birth weight in the first trimester included: maternal age, BMI, blood pressure, PAPP-A, BhCG and PIGF levels, fetal CRL and uterine artery PI.
\end{abstract}

Key words: birth weight; pre-existing diabetes; macrosomia; LGA; SGA

Ginekologia Polska 2019; 90, 7: 388-395

\section{INTRODUCTION}

Models of modern prenatal care aim at determining the risk of pregnancy-related complications during the first trimester. They also investigate any disorders related to the fetus growth. Numerous studies carried out on a large scale allowed for development of highly sensitive screening tests for fetal growth disorders based on using data from ultra- sound examinations (carried out between weeks 11-13+6 of pregnancy), accompanied by tests of mother blood serum.

Abnormal fetal growth, resulting in either large-for-gestational age (LGA), small-for-gestational age (SGA) or intrauterine growth restriction (IUGR), is a common fetal complication in high risk pregnancy [1, 2]. Therefore, early assessment of fetal growth and risk factors for inadequate 
fetal growth now gain considerable attention in the field of maternal-fetal medicine.

Maternal hyperglycemia is the main factor responsible for excessive fetal growth. Elevated maternal glucose levels intensify glucose transport to the fetus, which results in increased fetal production of insulin and insulin-like growth factors. This consequently leads to excessive development of adipose tissue in the developing fetus. Fetal growth undergoes strong genetic regulation but it is also modified by intrauterine environment (epigenetic factors) that determines the newborn final weight [3-5]. The most important risk factors for fetal macrosomia are: maternal diabetes and obesity, older age, gestational hypertension, and pregnancy lasting over 42 weeks. Excessive weight in the fetus causes several complications during prenatal development, and perinatal and postnatal life. In the perinatal period, LGA is associated with more frequent obstructed labors, higher percentage of pelvic floor muscle and maternal anal sphincter injuries, shoulder dystocia with consequent paralysis of the shoulder plexus, hypoxia and neonatal death.

Long-term observations of children with macrosomia show that as adults they are more prone to becoming overweight or obese and more often suffer from diabetes and cardiovascular disorders. In children with birth weight exceeding $4000 \mathrm{~g}$, higher frequency of insulin resistance, increased risk of metabolic syndrome, abnormal fasting blood glucose, and abnormal glucose tolerance in childhood are reported [6].

To reduce the risk of birth weight related complications, early identification of the risk group for fetal macrosomia and early elimination of potentially modifiable risk factors are important. The modern model of perinatal care called "an inverted pyramid"identifies early pregnancy as a period for perinatal risk assessment [7]. Thus, each pregnant woman is assigned to an appropriate risk group and specific interventions are commenced to reduce the identified risks. A risk analysis in early pregnancy also involves screening for abnormal fetal growth. An assessment model that draws upon data from a pregnancy history, nuchal translucency, and the levels of free $\beta$-human chorionic gonadotropin ( $\beta$-hCG) and pregnancy-associated plasma protein (PAPP-A) in the maternal serum between weeks 11-13 of pregnancy, identifies only about 35\% of women who would give birth to LGA newborns. Moreover, false positive rate is approximately $10 \%$ [8]. Further research in this field increased sensitivity and specificity of LGA screening by introducing new biomarkers (Inhibin A, selectin E, PLGF), and accounting for additional data obtained during ultrasound examination (pulsatility index PI, of the uterine artery). Despite that, majority of LGA cases remain undetected until actual delivery. Furthermore, we lack a prognostic tool that discriminates between a constitutionally large but healthy newborn and a neonate with "intrauterine obesity".

\section{Objectives}

The aim of our work was to assess the usefulness of maternal factors ultrasound and placental function parameters during early pregnancy as predictors of birth weight in populations of healthy pregnant women and women suffering from pregestational (type 1) diabetes.

\section{MATERIAL AND METHODS}

The prospective observational study included 97 healthy pregnant women (non-PGDM) and 160 pregnant women with pregestational diabetes (PGDM, type 1), all in singleton, non-malformed pregnancy, monitored on an outpatient basis during the pregnancy period at the Hospital Outpatient Clinic of the Podhalanski Specialist Hospital in Nowy Targ, Poland, or undergoing antenatal care in a tertiary referral unit of the Department of Obstetrics and Women's Diseases of the Poznan University of Medical Sciences, Poznan, Poland. All patients included in the study were informed about its purpose and scope and gave their written consent to participate.

Monitoring consisted of three follow-up visits during the pregnancy: between weeks 11 and $13+6$, and during weeks 20 and 30 . At the first visit, data were collected from each patient using a questionnaire. It requested information about the patient's age, BMI, reproductive history and comorbidities. It particularly focused on diabetes and its type, age of the patient at onset, pregnancy-induced hypertension, pre-eclampsia in previous pregnancies, and smoking.

The study, carried out between weeks 11 and $13+6$ of pregnancy, aimed at assessing fetal anatomy and evaluating markers of chromosomal aberrations using the recommendations of the Polish Society of Gynecologists and Obstetricians, Ultrasonography Division (crown rump length - CRL, biparietal diameter - BPD, nuchal translucency — NT, nasal bone - NB, ductus venosus - DV), and uterine artery blood flow parameters (UtA PI — pulsatility index). Blood samples collected during the visits were centrifuged, aliquoted and transported to the ISO 9000 accredited Central Laboratory of Clinical Gynecology and Obstetrics Hospital in Poznań, where PIGF and PAPP-A protein concentrations, and $\beta-\mathrm{hCG}$, PIGF and PAPP-A serum levels were determined in an immunofluorometric assay and DELFIA Xpress analyzer. $\beta$-hCG values were assessed using monoclonal antibodies labeled with a ruthenium complex.

We defined birth weight above the $90^{\text {th }}$ percentile for a gestational age and neonatal sex at delivery as a large-for-gestational age newborn (LGA). Birth weight below the $10^{\text {th }}$ percentile for a gestational age at delivery and neonatal sex were defined as a small-for-gestational age newborn (SGA). Percentiles were calculated referring to the non- PGDM subgroup in the study.

Statistical analysis was carried out using SPSS for Windows 14.0.0 (SPSS Inc. Chicago, USA) and MedCalc Statisti- 
cal Software, version 18.2.1 (MedCalc Software bvba, Ostend, Belgium; http://www.medcalc.org; 2018). Data were checked for normality and then appropriate parametric or nonparametric tests were used to check for differences between the variables studied in the PGDM and non-PGDM subgroups. Multiple regression models were built to identify predictors for the birth weight in the entire cohort, and for both researched groups. We used logistic regression and ROC analysis to identify predictors of abnormal birth weight, defined as large-for-gestational age or small-for-gestational age. Variables were presented as mean \pm standard deviation or median. $\mathrm{P}<0.05$ was considered statistically significant.

\section{RESULTS}

Table 1 summarizes the characteristics of the study groups. Of the entire cohort, $9.7 \%$ of participants had pregnancy-related hypertensive disorders, 9.7\% reported smoking during pregnancy, $30.7 \%$ were overweight or

\begin{tabular}{|c|c|c|c|c|c|c|c|}
\hline Variables & Patient group & Mean & Standard deviation (SD) & Median & Min & Max & Mann-Whitney Test \\
\hline \multirow{2}{*}{ Age [years] } & PGDM & 29.8 & 4.7 & 29.3 & 19.4 & 44.5 & \multirow{2}{*}{$p=0.030$} \\
\hline & non-PGDM & 28.5 & 5.3 & 27.0 & 19.0 & 41.0 & \\
\hline \multirow{2}{*}{ Height $[\mathrm{cm}]$} & PGDM & 166.0 & 6.3 & 165.0 & 153.0 & 186.0 & \multirow{2}{*}{$p=0.569$} \\
\hline & non-PGDM & 165.2 & 6.0 & 165.0 & 150.0 & 176.0 & \\
\hline \multirow{2}{*}{ Weight [kg] } & PGDM & 65.3 & 14.6 & 62.0 & 47.0 & 124.0 & \multirow{2}{*}{$p=0.227$} \\
\hline & non-PGDM & 66.2 & 12.4 & 62.9 & 44.6 & 99.0 & \\
\hline \multirow{2}{*}{ BMI } & PGDM & 23.7 & 5.1 & 22.6 & 16.7 & 47.8 & \multirow{2}{*}{$p=0.161$} \\
\hline & non-PGDM & 24.3 & 4.7 & 23.0 & 17.6 & 39.0 & \\
\hline \multirow{2}{*}{ Systolic BR } & PGDM & 112.3 & 14.6 & 111.9 & 80.0 & 150.8 & \multirow{2}{*}{$p=0.003$} \\
\hline & non-PGDM & 107.6 & 12.8 & 105.0 & 80.0 & 145.0 & \\
\hline \multirow{2}{*}{ Diastolic BP } & PGDM & 70.6 & 9.9 & 70.0 & 50.0 & 97.0 & \multirow{2}{*}{$p=0.006$} \\
\hline & non-PGDM & 67.3 & 9.7 & 65.0 & 50.0 & 90.0 & \\
\hline \multirow{2}{*}{ Mean BP } & PGDM & 84.5 & 11.0 & 83.3 & 60.0 & 112.9 & \multirow{2}{*}{$p=0.005$} \\
\hline & non-PGDM & 80.7 & 9.9 & 79.2 & 60.0 & 106.7 & \\
\hline \multirow{2}{*}{$\begin{array}{l}\text { Right UtA PI } \\
\text { (I trimester) }\end{array}$} & PGDM & 1.55 & 0.63 & 1.44 & 0.46 & 3.57 & \multirow{2}{*}{$p=0.426$} \\
\hline & non-PGDM & 1.60 & 0.58 & 1.49 & 0.57 & 3.49 & \\
\hline \multirow{2}{*}{$\begin{array}{l}\text { Left UtA PI } \\
\text { (I trimester) }\end{array}$} & PGDM & 1.53 & 0.60 & 1.52 & 0.45 & 3.43 & \multirow{2}{*}{$p=0.455$} \\
\hline & non-PGDM & 1.55 & 0.47 & 1.51 & 0.53 & 2.82 & \\
\hline \multirow{2}{*}{ Low UtAPI } & PGDM & 1.29 & 0.49 & 1.23 & 0.45 & 2.89 & \multirow{2}{*}{$p=0.225$} \\
\hline & non-PGDM & 1.20 & 0.40 & 1.17 & 0.40 & 2.64 & \\
\hline \multirow{2}{*}{ High UtAPI } & PGDM & 1.79 & 0.62 & 1.77 & 0.56 & 3.57 & \multirow{2}{*}{$p=0.001$} \\
\hline & non-PGDM & 2.10 & 0.67 & 1.98 & 0.90 & 3.98 & \\
\hline \multirow{2}{*}{ Mean UtAPI } & PGDM & 1.54 & 0.51 & 1.52 & 0.55 & 2.99 & \multirow{2}{*}{$p=0.469$} \\
\hline & non-PGDM & 1.59 & 0.44 & 1.52 & 0.85 & 2.88 & \\
\hline \multirow{2}{*}{ CRL [mm] } & PGDM & 65.1 & 8.3 & 64.0 & 48.0 & 83.0 & \multirow{2}{*}{$p=0.186$} \\
\hline & non-PGDM & 63.4 & 10.9 & 64.5 & 45.0 & 86.0 & \\
\hline \multirow{2}{*}{ NT [mm] } & PGDM & 1.38 & 0.33 & 1.40 & 0.70 & 2.50 & 00001 \\
\hline & non-PGDM & 2.49 & 8.40 & 1.70 & 1.00 & 83.00 & $p<0.001$ \\
\hline$\beta$-hCG & PGDM & 53.0 & 56.0 & 42.7 & 8.3 & 522.2 & $n=0023$ \\
\hline $\mathrm{U} / \mathrm{I}$ & non-PGDM & 41.5 & 31.2 & 33.1 & 6.3 & 232.8 & מכנט \\
\hline PAPP_A III & PGDM & 3.07 & 1.98 & 2.77 & 0.22 & 9.43 & $n-0689$ \\
\hline 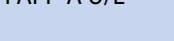 & non-PGDM & 3.26 & 3.94 & 2.44 & 0.67 & 36.45 & (5) \\
\hline C5 & PGDM & 43.5 & 15.0 & 39.9 & 1.7 & 100.0 & 0001 \\
\hline [LUT [Ng/L] & non-PGDM & 34.0 & 14.5 & 30.4 & 13.6 & 90.8 & $p<0.001$ \\
\hline Dive & PGDM & 3388 & 596 & 3415 & 980 & 4660 & $0-0.0-10$ \\
\hline Dil tir vergmit & non-PGDM & 3314 & 512 & 3350 & 1660 & 4600 & 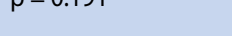 \\
\hline
\end{tabular}


obese (compared with $13.2 \%$ of our subgroup). In the PGDM subgroup, $10.1 \%$ of participants had vascular complications (retinopathy, and/or nephropathy).

There were no statistically significant differences between patients with pre-gestational diabetes and nondiabetic participants regarding height, body weight, and BMI, $\mathrm{Pl}$ in the right and left uterine artery, the lowest and average $\mathrm{PI}$ value of the uterine artery, crown rump length (CRL), PAPP-A levels or child birth weight.

Data concerning correlations between the fetal and maternal parameters collected during early pregnancy and neonatal body weight are summarized in Table 2 .

We found that in the non-PGDM subgroup birth weight significantly correlated only with maternal BMI, whereas among the diabetic patients significant correlations were confirmed for maternal age, CRL, vascular flow and concentration of placental proteins.
In order to investigate the influence of selected early pregnancy parameters on birth weight in the entire cohort, stepwise regression was built. The best-fit model for the entire cohort is presented in Table 3.

After investigating for early pregnancy maternal and fetal parameters, we found that PAPPA concentrations, early pregnancy maternal body weight and hypertensive status remained statistically significant predictors of birth weight across the entire study group.

In a separate analysis, we looked for predictors of abnormal fetal growth, defined as either LGA or SGA. In our study group, we had 39 cases of LGA out of 226 mother-infant pairs (17.2\%, no data available for 31 patients). After investigating for fetal and maternal parameters, we confirmed that only mean UtAPI remained a statistically significant predictor of LGA (Tab. 4). However, we also identified several independent predictors of LGA in our cohort that included PIGF, NT, and CRL (Fig. 1 A-D).

\begin{tabular}{|c|c|c|c|c|c|c|}
\hline \multirow{2}{*}{ Pairs of correlated variables } & \multicolumn{2}{|c|}{ STUDY GROUP } & \multicolumn{2}{|l|}{ PGDM } & \multicolumn{2}{|c|}{ non-PGDM } \\
\hline & $\mathbf{R}$ & p & $\mathbf{R}$ & $p$ & $\mathbf{R}$ & p \\
\hline Birth weight [g] \& Age & -0.129 & 0.049 & -0.165 & 0.044 & -0.100 & 0.356 \\
\hline Birth weight $[g]$ \& BMI & 0.076 & 0.247 & 0.014 & 0.861 & 0.224 & 0.039 \\
\hline Birth weight [g] \& systolic BP & 0.021 & 0.747 & -0.040 & 0.626 & 0.067 & 0.543 \\
\hline Birth weight [g] \& diastolic BP & -0.028 & 0.674 & -0.076 & 0.358 & 0.037 & 0.738 \\
\hline Birth weight [g] \& Mean BP & -0.002 & 0.978 & -0.055 & 0.508 & 0.047 & 0.666 \\
\hline Birth weight [g] \& right UtAPI & -0.065 & 0.327 & -0.109 & 0.194 & 0.032 & 0.770 \\
\hline Birth weight [g] \& left UtAPI & -0.168 & 0.014 & -0.177 & 0.033 & -0.131 & 0.282 \\
\hline Birth weight [g] \& low UtAPI & -0.087 & 0.189 & -0.131 & 0.116 & -0.010 & 0.925 \\
\hline Birth weight [g] \& high UtAPI & -0.131 & 0.048 & -0.167 & 0.044 & -0.022 & 0.839 \\
\hline Birth weight [g] \& mean UtAPI & -0.128 & 0.052 & -0.161 & 0.054 & -0.062 & 0.570 \\
\hline Birth weight [g] \& CRL [mm] & 0.085 & 0.194 & 0.164 & 0.046 & -0.053 & 0.631 \\
\hline Birth weight [g] \& NT [mm] & 0.006 & 0.930 & 0.049 & 0.554 & 0.042 & 0.704 \\
\hline Birth weight [g] \& B-hCG IU/L & -0.043 & 0.516 & -0.080 & 0.336 & 0.009 & 0.935 \\
\hline Birth weight [g] \& PAPP-A U/L & 0.119 & 0.072 & 0.258 & 0.002 & -0.163 & 0.140 \\
\hline Birth weight [g] \& PLGF [pg/L] & 0.187 & 0.006 & 0.284 & 0.001 & 0.042 & 0.734 \\
\hline
\end{tabular}

$\mathrm{p}$ - level of significance; $\mathrm{R}$ - Spearman's rank correlation coefficient

Table 3. Predictors of birth weight in the entire cohort - multiple regression

\begin{tabular}{|c|c|c|c|c|c|}
\hline \multicolumn{6}{|c|}{$\begin{array}{l}\text { Summary of dependent variable regression: Birth weight }[g] \\
R=0.341 R^{2}=0.116 \text { correct; } R^{2}=0.102 ; F(5.185)=3.9103 ; p<0.0001 ; \text { standard estimation error: } 560.279\end{array}$} \\
\hline \multirow{2}{*}{ Regression model } & \multirow{2}{*}{$\begin{array}{l}\text { Standardized coefficients } \\
\text { beta }\end{array}$} & \multicolumn{2}{|c|}{ Non-standardized coefficients } & \multirow{2}{*}{$t(185)$} & \multirow{2}{*}{$\mathbf{p}$} \\
\hline & & B & SE & & \\
\hline Intercept & & 2257.217 & 250.085 & 9.027 & $p<0.0001$ \\
\hline PAPPA serum level & 0.274 & 79.907 & 21.711 & 3.680 & $p<0.0001$ \\
\hline Maternal body weight & 0.309 & 13.397 & 3.341 & 4.010 & $p<0.0001$ \\
\hline Hypertensive disorders of pregnancy YES & -0.149 & -287.703 & 140.234 & -2.052 & $p=0.042$ \\
\hline
\end{tabular}


In the entire cohort we had 24 cases of SGA $(10.6 \%$, no data available for 31 patients). None of the analyzed variables significantly predicted birth weight below the $10^{\text {th }}$ percentile.

In a separate analysis of the subgroups, we identified several predictors of birth weight in PGDM subgroup that remained statistically significant after adjustment: the moth-
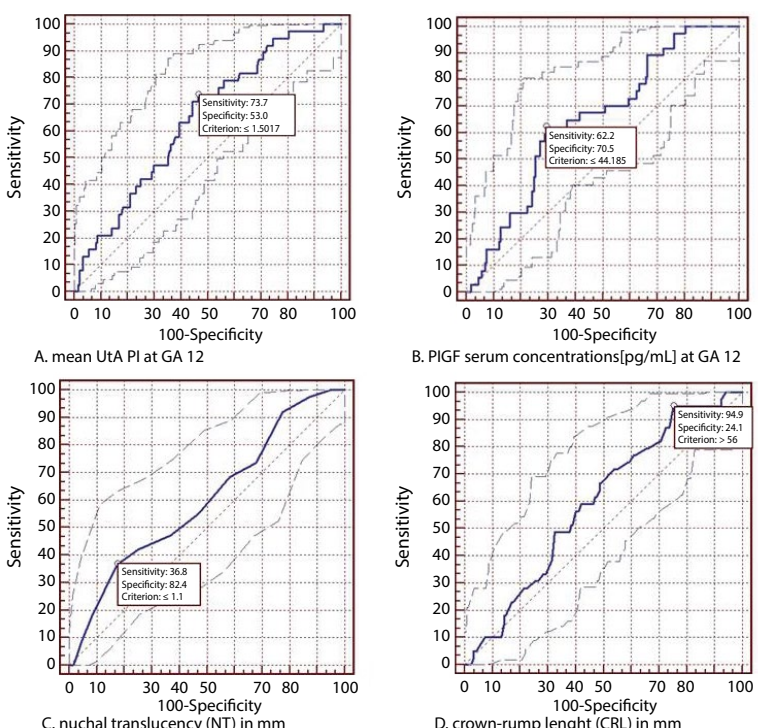

Figure 1. Independent predictors of LGA in the whole cohort er's age, gestational age at examination, glycemic levels below a pathological limit, and duration of diabetes. Data from the multiple regression model are presented in Table 5 .

To identify predictive factors for excessive fetal growth in diabetic pregnancy, we built a model of logistic regression with LGA as a dependent variable. After adjustments for fetal and maternal confounders, mean UtAPI and PAPPA serum level remained as statistically significant predictors of LGA in this subgroup (Tab. 6). However, we also identified CRL and PLGF as independent LGA predictors (Fig. 2 A-B).

InPGDM subgroup, wehad 17 cases of SGA. After adjusting for confounders, maternal PLGF serum level was found to be a statistically significant predictor of low birth weight but with a minimal actual impact on this outcome (Tab. 7). We also noted that PAPPA serum levels independently predicted SGA in this cohort (Fig. 3 A-B).

In a separate analysis of non-PGDM subgroup, none of the parameters contributed significantly to the risk of LGA.

\section{DISCUSSION}

Early detection of pregnant women with high risk of fetal growth disorders allows for closer monitoring of these patients, possible modification of risk factors and informed decisions concerning the mode of delivery. Recent research in this field abounds in algorithms based on data obtained during the first trimester screening, supplemented with

Table 4. Predictors of LGA in the entire cohort - logistic regression

\begin{tabular}{|c|c|c|c|c|}
\hline Logistic regression FSTEP model & Regression coefficient B & p & Odds ratio (OR) & 95\% confidence interval for OR \\
\hline Intercept & 0.64 & $p=0.329$ & 1.90 & \\
\hline mean UtAPI & -1.496 & $p=0.001$ & 0.22 & 0.09 \\
\hline
\end{tabular}

Table 5. Predictors of birth weight in PGDM patients - multiple regression

\begin{tabular}{|c|c|c|c|c|c|}
\hline \multicolumn{6}{|c|}{$\begin{array}{l}\text { Summary of dependent variable regression: Birth weight }[\mathrm{g}] \\
\mathrm{R}=0.454 ; \mathrm{R} 2=0.206 \text { Correct; } \mathrm{R} 2=0.182 ; \mathrm{F}(6.133)=6.2581 ; \mathrm{p}<0.00001 \text {; standard estimation error: } 539.602\end{array}$} \\
\hline \multirow{2}{*}{ Regression model } & \multirow{2}{*}{$\begin{array}{l}\text { Standardized coefficients } \\
\text { beta }\end{array}$} & \multicolumn{2}{|c|}{ Non-standardized coefficients } & \multirow{2}{*}{$t(185)$} & \multirow{2}{*}{$\mathbf{p}$} \\
\hline & & B & SE & & \\
\hline Intercept & & 860.804 & 1220.459 & 0.705 & $p=0.482$ \\
\hline PAPP-A U/L & 0.353 & 103.3065 & 23.036 & 4.487 & $p<0.0001$ \\
\hline$\beta$-hCG IU/L & -0.197 & -2.015 & 0.791 & -2.546 & $p=0.012$ \\
\hline Maternal height $[\mathrm{cm}]$ & 0.196 & 18.402 & 7.251 & $2, .538$ & $p=0.012$ \\
\hline Diastolic BP at the examination & -0.182 & -10.801 & 4.702 & -2.297 & $p=0.023$ \\
\hline
\end{tabular}

Table 6. Predictors of LGA in PGDM patients — logistic regression

\begin{tabular}{|c|c|c|c|c|c|}
\hline Logistic regression FSTEP model & Regression coefficient B & $\mathbf{p}$ & Odds ratio (OR) & \multicolumn{2}{|c|}{$\mathbf{9 5} \%$ confidence interval for OR } \\
\hline Intercept & -0.379 & $p=0.643$ & 0.684 & & \\
\hline PAPP-A U/L & 0.205 & $p=0.043$ & 1.23 & 1.01 & 1.50 \\
\hline Mean UtAPI & -1.162 & $p=0.018$ & 0.313 & 0.12 & 0.82 \\
\hline
\end{tabular}




\begin{tabular}{|l|l|l|l|l|l|}
\hline \multicolumn{2}{|l}{ Table 7. Predictors of SGA in PGDM patients - logistic regression } \\
\hline Logistic regression FSTEP model & Regression coefficient B & $\mathbf{p}$ & Odds ratio (OR) & $\mathbf{9 5 \%}$ confidence interval for OR \\
\hline Intercept & 0.147 & $p=0.880$ & 0.95 & & 0.995 \\
\hline PLGF [pg/L] & -0.065 & $p=0.030$ & 1.16 & 0.901 & \\
\hline
\end{tabular}
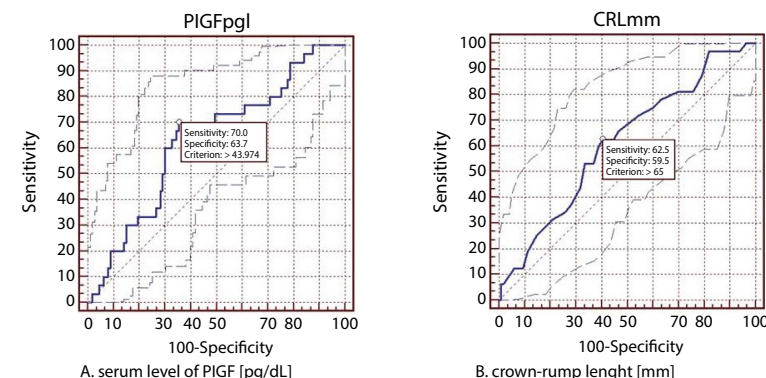

Figure 2. Independent predictors of LGA in PGDM patients
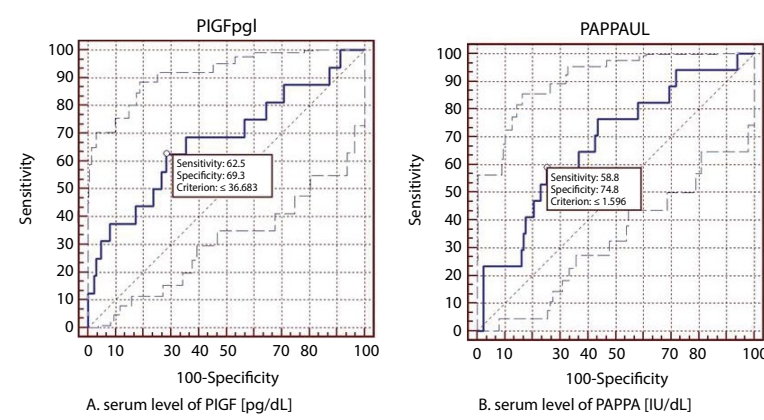
B. serum level of PAPPA [IU/dL]

Figure 3. Predictors of SGA in PGDM patients

additional data from ultrasound examination and on plasma protein concentrations.

In 2013, Papastefanou et al. performed prenatal examinations in 702 first trimester patients. They created a diagnostic model in which significant, independent SGA predictors included patient height, multiparity, smoking, assisted reproduction, CRL, NT and PAPP-A and $\beta$-hCG levels. They also found that the weight and height of the pregnant women, cigarette smoking, and CRL and NT levels were significant, independent predictors of LGA. Sensitivity of both models was relatively low - 48\% for LGA and 55\% for SGA [9]. The importance of the body mass index in LGA prediction was confirmed in another prospective screening study, based on examination of 41.577 pregnancies. Regression analysis showed a significant contribution from maternal BMI, in addition to maternal characteristics and obstetric history, in the prediction of subsequent delivery of small and large for gestational age neonates. The risk of LGA increased exponentially with increasing maternal BMI [10].

In 2016, Frick et al. published an observational study of a large cohort of pregnant women who were having fetal growth monitored throughout their pregnancies. The likelihood of developing LGA grew with increasing weight and height of the mothers, and decreased in tobaccosmoking and nulliparous women. Higher LGA risk was also found in patients with pre-gestational diabetes type 1, but dropped in patients with chronic hypertension. In multiparous women, LGA risk increased if LGA newborn was delivered before, and decreased in patients with previous gestational diabetes and short interval between consecutive pregnancies. The screening study was solely based on medical history data taken from 76.300, 54.999, 25.727 and 6.181 singleton pregnancies at 11-13, 19-24, 30-34, and 35-37 weeks' gestation, respectively. Screening by maternal factors at $11-13$ weeks predicted $32 \%, 44 \%$ and $60 \%$ of LGA $>95^{\text {th }}$ at false-positive rates (FPRs) of $5 \%, 10 \%$ and $20 \%$, respectively. With the addition of fetal biometry, the detection rates improved to $37 \%, 51 \%$ and $68 \%$ at $19-24$ weeks, $50 \%, 65 \%$ and $81 \%$ at $30-34$ weeks and $60 \%$, $73 \%$ and $85 \%$ at $35-37$ weeks at FPRs of $5 \%, 10 \%$ and $20 \%$, respectively. The addition of biomarkers did not improve the detection rates achieved when screening by a combination of maternal history and fetal biometry [11].

An LGA prediction algorithm similar to that mentioned above was used in an Italian study of 72 pregnant women between weeks 11-14 of pregnancy. The LGA prediction used historical data and PAPP-A concentration levels. Maternal growth, age, smoking, assisted reproduction and PAPP-A levels were found to be important, independent predictors of LGA [12]. In another study, Gonzalez et al. conducted a prenatal examination of 2097 pregnant women, including a Doppler study of the uterine artery in the first trimester and an assessment of fetal growth and the uterine artery Doppler in the second trimester. The study algorithm based on maternal history, PAPP-A protein concentrations, and $\beta-h C G$, $N T$, and $P I$ values in the uterine arteries, allowed for identification of $30.2 \%$ of LGA cases, with a false positive rate of $10 \%$. When data from the second trimester were added, the sensitivity increased to $56.2 \%$ with FPR of $20 \%$ [13]. Another biomarker that turned out useful in the prediction of macrosomic neonates was adiponectin. Examination of 350 cases showed that in the macrosomic group the median serum adiponectin was significantly lower than in the non-macrosomic controls. A detection rate of macrosomia, based on maternal characteristics and obstetric history was $34.6 \%$ with false positive rate of 
$10 \%$. Inclusion of adiponectin to this algorithm increased the detection rate to $38.2 \%$ [14].

Boucoiran at al. used only the parameters from the first trimester screening for aneuploidies to predict the birth weight. They examined 4110 patients in a singleton pregnancy. NT was significantly higher in LGA group as compared with the unaffected group but biomarkers (PAPPA and BhCG) were at the same levels in both groups. After controlling for gestational age, maternal weight, smoking status, ethnicity, and fetal sex, first-trimester markers contributed to the prediction of birth weight in a multiple linear model but did not significantly improve the prediction of LGA as compared with maternal characteristics alone [15].

There are few studies referring to growth disorders in the fetuses of mothers with pre-gestational diabetes, which used plasma protein concentrations and data from prenatal ultrasound examination between weeks 11-14 of pregnancy in their algorithm. The most frequently assessed predictors of growth disorders in such fetuses were fasting glycemia and gyrated hemoglobin concentrations. Some information about the risk of LGA and fetal macrosomy in pregnancies complicated by a metabolic syndrome and obesity can be found in the first trimester screening carried out by Migda et al. in 123 Caucasian patients with the metabolic syndrome. In that study, BMl above 25.5 was found an important risk factor for excessive fetal weight. Mother blood glucose and concentrations of adiponectin and soluble E-selectin were predictive of LGA and fetal macrosomia. The mother weight of or exceeding $67 \mathrm{~kg}$ in the first trimester showed high sensitivity and specificity in detecting LGA and macrosomia [16]. However, that study investigated a completely different population than ours.

Based on our cohort, we can conclude that placentation and placental function during early pregnancy strongly affect fetal growth, particularly in PGDM complicated pregnancy. Importantly, early pregnancy vascular function seems to be even more crucial for fetal growth than maternal pre-pregnancy glycemic control. The latter needs to be achieved if a reduced risk of fetal malformation or miscarriage is aimed at, while uteroplacental capacity ensures adequate transfer of oxygen and nutrients.

Our observations confirm that genetic potential (seen as $\mathrm{CRL}$ ), uteroplacental vascular capacity (measured as UtAPI), and placental function (measured as serum levels of specific placental proteins) shape the growth trajectory for fetuses. Those predictors, supported by data commonly available from the patient's history (age, BMI, and blood pressure) can be used in the algorithm for the first trimester. In our study, birth weight of the newborn negatively correlated with the age of the mother, her diastolic blood pressure, $\mathrm{PI}$ of the uterine artery and BhCG protein levels. PIGF and PAPPA-A protein levels and maternal early-pregnancy BMI correlated positively with birth weight of the newborn. Importantly, using an LGA prediction algorithm in the first trimester of pregnancies complicated by PGDM, we are able to estimate the baseline risk of fetal growth disorders in the diabetic population before the maternal glycemia affected the fetal growth trajectory.

\section{CONCLUSIONS}

1. LGA diagnosis in the first trimester of pregnancy allows for the selection and modification of some risk factors and closer monitoring of endangered fetuses throughout the pregnancy, with emphasis on the perinatal period.

2. Parameters with confirmed usefulness in the prediction of the birth weight in the first trimester include maternal age, BMI, blood pressure, PAPP-A, BhCG and PIGF values; $\mathrm{CRL}$ measurement and uterine artery PI values.

\section{Authors' contributions}

1. Anna Gąsiorowska — designed the experiment, collected the material, drafted the manuscript and researched the literature.

2. Agnieszka Zawiejska - analyzed and interpreted the results, critically revised the manuscript.

3. Piotr Dydowicz - collected the material.

4. Ewa Wender-Ożegowska - critically revised the manuscript.

5. Grzegorz Poprawski - collected the material.

6. Kinga Toboła-Wróbel - analyzed and interpreted the data.

7. Katarzyna Ziółkowska - collected the material and carried out biochemical tests.

8. Marek Pietryga - designed the experiment, proofread, verified and finally approved the manuscript.

\section{Funding}

The research reported in the manuscript was not financed or co-financed by any R\&D centre, association or any other institution or entity. The authors did not receive any research grant.

\section{Conflict of interests}

The authors declare no conflicts of interest. No financial remuneration was received relative to the technologies and concepts presented in the manuscript.

\section{REFERENCES}

1. Bomba-Opoń D, Drews $K$, Huras $H$, et al. Polish Gynecological Society Recommendations for Labor Induction. Ginekol Pol. 2017; 88(4): 224-234, doi: 10.5603/GP.a2017.0043, indexed in Pubmed: 28509326.

2. Ropacka-Lesiak M. Wewnątrzmaciczne ograniczenie wzrastania płodu. In: Bręborowicz GH. ed. Położnictwo Tom 2: Medycyna matczynopłodowa. PZWL, Warszawa 2012: 105-117. 
3. Baschat AA, Galan IH, Gabbe SG. Wewnattrzmaciczne zahamowanie wzrastania płodu. In: Dębski R, Oszukowski P. ed. Położnictwo. Ciąża prawidłowa i powikłana. Elsevier Urban \& Partner, Wrocław 2014: 146-181.

4. Hirnle L, Kowalska M, Petrus A, et al. [The analysis of risk factors for fetal macrosomia and the complications in the course of pregnancy and delivery of macrosomic baby]. Ginekol Pol. 2007; 78(4): 280-283, indexed in Pubmed: 17621988.

5. Swierzewska P, Kosiński M, Wójcik M, et al. Family, anthropometric and biochemical factors affecting birth weight of infants born to GDM women. Ginekol Pol. 2015; 86(7): 499-503, indexed in Pubmed: 26376526.

6. Ornoy A. Prenatal origin of obesity and their complications: Gestational diabetes, maternal overweight and the paradoxical effects of fetal growth restriction and macrosomia. Reprod Toxicol. 2011; 32(2): 205-212, doi: 10.1016/j.reprotox.2011.05.002, indexed in Pubmed: 21620955.

7. Nicolaides $\mathrm{KH}$. A model for a new pyramid of prenatal care based on the 11 to 13 weeks' assessment. Prenat Diagn. 2011; 31(1): 3-6, doi: 10.1002/pd.2685, indexed in Pubmed: 21210474.

8. Poon LCY, Karagiannis G, Stratieva V, et al. First-trimester prediction of macrosomia. Fetal Diagn Ther. 2011; 29(2): 139-147, doi: 10.1159/000318565, indexed in Pubmed: 20798483.

9. Papastefanou I, Souka AP, Pilalis A, et al. First trimester prediction of small- and large-for-gestation neonates by an integrated model incorporating ultrasound parameters, biochemical indices and maternal characteristics. Acta Obstet Gynecol Scand. 2012; 91(1): 104-111, doi: 10.1111/j.1600-0412.2011.01271.x, indexed in Pubmed: 21895614.
10. Syngelaki A, Bredaki FE, Vaikousi $E$, et al. Body mass index at 11-13 weeks' gestation and pregnancy complications. Fetal Diagn Ther. 2011; 30(4): 250-265, doi: 10.1159/000328083, indexed in Pubmed: 22067258.

11. Frick AP, Syngelaki A, Zheng M, et al. Prediction of large-for-gestational-age neonates: screening by maternal factors and biomarkers in the three trimesters of pregnancy. Ultrasound Obstet Gynecol. 2016; 47(3): 332-339, doi: 10.1002/uog.15780, indexed in Pubmed: 26446185.

12. Rossi A, Vogrig E, Ganzitti L, et al. Prediction of large-for-gestation neonates with first-trimester maternal serum PAPP-A. Minerva Ginecol. 2014; 66(5): 443-447, indexed in Pubmed: 24743523.

13. González González NL, Plasencia W, González Dávila E, et al. First and second trimester screening for large for gestational age infants. J Matern Fetal Neonatal Med. 2013; 26(16): 1635-1640, doi: 10.3109/14767058.2013.794779, indexed in Pubmed: 23668692.

14. Nanda S, Akolekar R, Sarquis R, et al. Maternal serum adiponectin at 11 to 13 weeks of gestation in the prediction of macrosomia. Prenat Diagn. 2011; 31(5): 479-483, doi: 10.1002/pd.2723, indexed in Pubmed: 21394735.

15. Boucoiran I, Djemli A, Taillefer C, et al. First-trimester prediction of birth weight. Am J Perinatol. 2013; 30(8): 665-672, doi: 10.1055/s-00321331023, indexed in Pubmed: 23283804.

16. Migda M, Migda MS, Migda B, et al. Maternal first trimester parameters in the prediction of excessive fetal growth in pregnant women with metabolic syndrome. J Physiol Pharmacol. 2017;68(6): 833-839, indexed in Pubmed: 29550795. 